\title{
SLIDING MODE OBSERVERS AND OBSERVABILITY SINGULARITY IN CHAOTIC SYNCHRONIZATION
}

\author{
L. BOUTAT-BADDAS, J. P. BARBOT, D. BOUTAT, AND R. TAULEIGNE
}

Received 29 September 2003 and in revised form 28 January 2004

We present a new secured data transmission based on a chaotic synchronization and observability singularity. For this, we adopt an approach based on an inclusion of the message in the system structure and we use a sliding mode observer for system with unknown input in order to recover the information. We end the paper with an example of chaotic system with an observability bifurcation. Moreover, this example highlights some benefits of the so-called step-by-step sliding mode observer.

\section{Introduction}

The main objective of this paper is to highlight the efficiency of mathematical tools such as normal form and bifurcation and also the efficiency of control theory method as sliding mode and observer design in the secure data transmission by the synchronization of chaotic systems.

Since the last decade (see [16]), by its application to secure communications, the synchronization of the chaotic systems drew great attention of several control theory researchers (for the sake of simplicity, synchronization here means a unidirectional synchronization). An interesting approach to chaos synchronization based on the concept of observer design has been proposed in [15] by Nijmeijer and Mareels. More precisely, consider the two systems

$$
\begin{aligned}
\dot{x} & =f(x), \\
\dot{z} & =f(z),
\end{aligned}
$$

where $x \in \mathbb{R}^{n}, z \in \mathbb{R}^{n}$, and $f: \mathbb{R}^{n} \rightarrow \mathbb{R}^{n}$ is a nonlinear vector field.

We say that systems (1.1) and (1.2) are synchronized if

$$
e(t)=(z(t)-x(t)) \longrightarrow 0 \text { as } t \longrightarrow+\infty,
$$

where $e(t)$ represents the synchronization error (for a general definition, see [6]). 


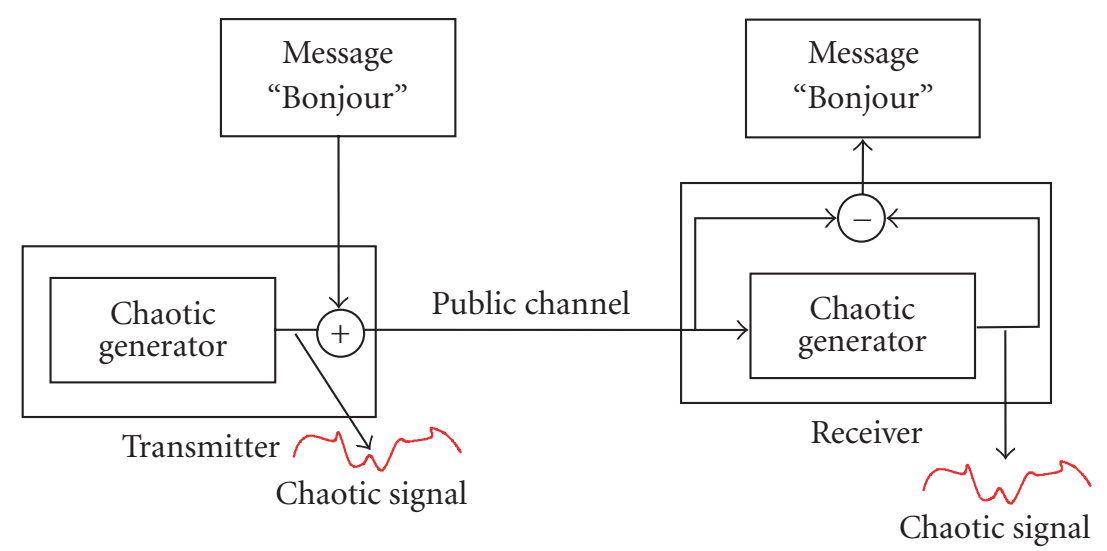

Figure 1.1. Additive chaos masking.

An observer is a dynamical system designed to be driven by the output of another system (transmitter) and having the property that the state of the observer converges to the state of the original system. More precisely, given system (1.1) with output $y=h(x) \in$ $\mathbb{R}^{n}$, the dynamic system

$$
\dot{\hat{x}}=f(\hat{x}, y)
$$

is said to be an observer for system (1.1) if $\hat{x}(t)$ converges to the state $x(t)$ as $t \rightarrow+\infty$ for any initial conditions $x(0)$ and $\hat{x}(0)$ in a considered domain.

From [15], many techniques have been developed to design an observer for a chaotic system. Under some appropriate assumptions, as, for example, conditions of linearization by output injection form $[13,14]$ or conditions for obtaining a generalized Hamiltonian form [19], this design may be done without any difficulty. Often, the cryptographic techniques are based on masking the information by adding it to a chaotic signal on the transmission line (see Figure 1.1) [7]. Information transfer, in a secured way, by the addition of the signal carrying the information, is possible, thanks to the synchronization of two copies of a chaotic system in the sense that their state trajectories tend asymptotically to be identical when one system is suitably driven by the other. One of the disadvantages of additive chaos masking of chaotic secure communication schemes is the low efficiency of cannel usage.

In this paper, we adopt an approach based on including the information in the chaotic system structure (transmitter). Consequently, information does not evolve a separate form of chaos but will be constantly handled by the system dynamics. This approach is called chaotic parameter modulation [24] (shown in Figure 1.2).

In [24], in order to synchronize two chaotic systems and to decipher a confidential message, Yang and Chua used an adaptive controller. The conception of such controller is based on the singular perturbation methods (see $[12,20])$. In the singular perturbation 


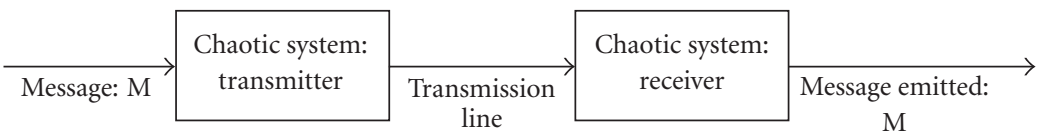

Figure 1.2. The chaotic parameter modulation.

theory (see [12]), various speeds of evolution (different time scales) are exploited. Consequently, it is requested then that the message behavior is slower than the system dynamics in [24].

The method proposed in this paper consists of an observer design with unknown input (here the message), allowing to reconstitute the confidential message from the only information passing to the receiver by the transmitter, and this is for a message without dynamical assumption. However, a question settles, namely, how to design an observer for the nonlinear systems with observability singularity?

Historically, in nonlinear control theory, the problem of a nonlinear observer design with linearization of the observation error dynamic for a class of nonlinear systems, called the output injection form, has been investigated. Unfortunately, the geometric conditions in order to obtain this form are very often too restrictive with respect to the system considered (for more details, see $[2,4,13,14,17]$ ).

In this paper, we propose an observer design for a system with unknown input and which has an observability singularity. To overcome such difficulties and in order to increase the busy band of the message and take into account the structural change of the system, we use a step-by-step sliding mode observer, which is a variable structure observer able to take into account some structural singularity and also is robust in the parameter variations, if an observability matching condition is verified.

For this, we have to recall and give some particular concepts of observer for a system with unknown input. In the same way of thinking, in [4], we have introduced the same concept for observability (observability singularity) and we recover naturally some properties as universal inputs [8], resonant terms [22], and so on.

\section{Structure of the observer for chaos synchronization}

The linearization by output injection (see $[13,14]$ ) is a usual tool to design an observer and consequently to resolve the synchronization problem. This approach to synchronization is valid if the nonlinearity of a system depends only on the output. However, by considering another output, it is possible to design a step-by-step sliding mode observer (see $[2,17]$ ) in spite of the fact that linearization by output injection is not possible (we highlight the efficiency of the sliding mode observer and more particularly of the stepby-step observer with respect to the observability matching condition. This condition is used in order to ensure the data transmission).

In this section, we design an observer for the well-known Chua circuit (Figure 2.1). This circuit is a simple oscillator which exhibits a variety of bifurcations and chaos. The circuit contains a linear resistor $\left(R, R_{0}\right)$, a single nonlinear resistor $\left(f\left(v_{1}\right)\right)$, and three linear energy-storage components: an inductor $(L)$ and two capacitors $\left(C_{1}, C_{2}\right)$. The state 

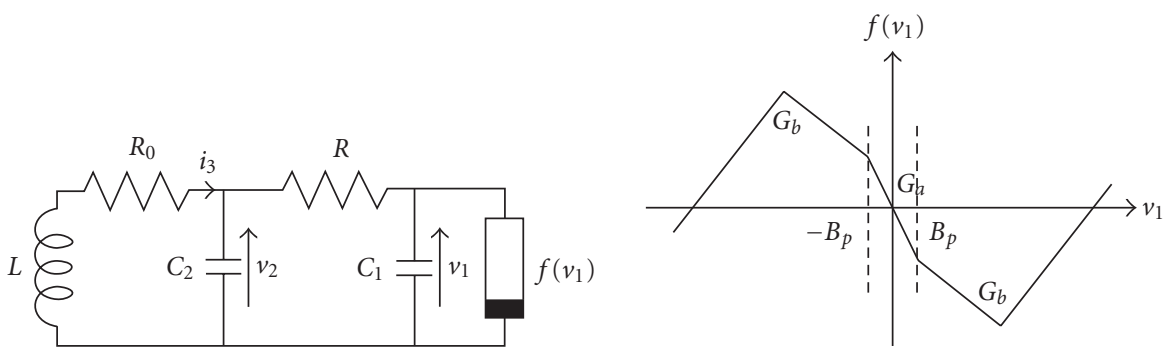

Figure 2.1. Chua circuit.

equations for the circuit are as follows:

$$
\begin{aligned}
\frac{d v_{1}}{d t} & =\frac{1}{C_{1}}\left(\frac{v_{2}-v_{1}}{R}-f\left(v_{1}\right)\right), \\
\frac{d v_{2}}{d t} & =\frac{1}{C_{2}}\left(\frac{v_{1}-v_{2}}{R}+i_{3}\right), \\
\frac{d i_{3}}{d t} & =\frac{1}{L}\left(-v_{2}-R_{0} i_{3}\right)
\end{aligned}
$$

with $f\left(v_{1}\right)=G_{b} v_{1}+0.5\left(G_{a}-G_{b}\right)\left(\left|v_{1}+E\right|-\left|v_{1}-E\right|\right)$.

Setting $x_{1} \triangleq v_{1}, x_{2} \triangleq v_{2}, x_{3} \triangleq i_{3}$, and $x \triangleq\left(x_{1}, x_{2}, x_{3}\right)^{T}$, we obtain

$$
\begin{aligned}
\frac{d x_{1}}{d t} & =\frac{-1}{C_{1} R}\left(x_{1}-x_{2}\right)+\frac{f\left(x_{1}\right)}{C_{1}}, \\
\frac{d x_{2}}{d t} & =\frac{1}{C_{2} R}\left(x_{1}-x_{2}\right)+\frac{x_{3}}{C_{2}}, \\
\frac{d x_{3}}{d t} & =\frac{-1}{L}\left(x_{2}+R_{0} x_{3}\right) .
\end{aligned}
$$

2.1. Nonlinearity of Chua circuit depends only on the output. The problem of a nonlinear observer design with linearization of the observation error dynamics for a class of nonlinear systems, called the output injection form, has been investigated. Some necessary and sufficient conditions to obtain such a form are given in [13]. From this form, it is "easy" to design an observer. Unfortunately, the geometric conditions to obtain this form are very restrictive.

2.1.1. Linearizable error dynamics case. We choose as output $y$ the state $x_{1}$. It is clear that the system is globally weakly observable (see [10]) and linearizable by output injection. Then, there exist many observers for this system. According to our knowledge, the first classical one was proposed by Parlitz in [16]: 


$$
\begin{aligned}
\frac{d \hat{x}_{1}}{d t} & =\frac{1}{C_{1}}\left(\frac{\hat{x}_{2}-\hat{y}}{R}-f(\hat{y})\right), \\
\frac{d \hat{x}_{2}}{d t} & =\frac{1}{C_{2}}\left(\frac{y-\hat{x}_{2}}{R}+\hat{x}_{3}\right), \\
\frac{d \hat{x}_{3}}{d t} & =\frac{1}{L}\left(-\hat{x}_{2}-R_{0} \hat{x}_{3}\right), \\
y & =x_{1},
\end{aligned}
$$

where $\hat{x}=\left(\hat{x}_{1}, \hat{x}_{2}, \hat{x}_{3}\right)^{T}$ is the estimated state of $x$ and $\hat{y}$ is the estimated output. Since [15], the receiver design was changed and was closer to the observer design.

2.1.2. Step-by-step sliding mode observer. Throughout the paper, we will use a step-bystep sliding mode observer. This kind of observer is very useful and was developed for many reasons:

(i) to work with reduced observation error dynamics;

(ii) for a finite-time convergence for all components of the observable states;

(iii) robustness under parameter variations is possible if a specific condition (dual of the well-known matching condition) is verified.

To do this, we give hereafter such a kind of observer for system (2.2) with $y=x_{1}$ as output:

$$
\begin{aligned}
\frac{d \hat{x}_{1}}{d t} & =\frac{1}{C_{1}}\left(\frac{\hat{x}_{2}-y}{R}-f(y)\right)+\lambda_{1} \operatorname{sign}(y-\hat{y}), \\
\frac{d \hat{x}_{2}}{d t} & =\frac{1}{C_{2}}\left(\frac{y-\tilde{x}_{2}}{R}+\hat{x}_{3}\right)+E_{1} \lambda_{2} \operatorname{sign}\left(\tilde{x}_{2}-\hat{x}_{2}\right), \\
\frac{d \hat{x}_{3}}{d t} & =\frac{1}{L}\left(-\tilde{x}_{2}-R_{0} \tilde{x}_{3}\right)+E_{2} \lambda_{3} \operatorname{sign}\left(\tilde{x}_{3}-\hat{x}_{3}\right), \\
y & =x_{1},
\end{aligned}
$$

where the sign denotes the usual sign function, and the following conditions are satisfied: if $\hat{x}_{1}=x_{1}$, then $E_{1}=1$, else $E_{1}=0$, and if $\hat{x}_{2}=\tilde{x}_{2}$ and $E_{1}=1$, then $E_{2}=1$, else $E_{2}=0$. Moreover, by definition, we have the following auxiliary state:

$$
\begin{aligned}
& \tilde{x}_{2}=\hat{x}_{2}+E_{1} C_{1} R \lambda_{1} \operatorname{sign}(y-\hat{y}), \\
& \tilde{x}_{3}=\hat{x}_{3}+E_{2} C_{2} R \lambda_{2} \operatorname{sign}\left(\tilde{x}_{2}-\hat{x}_{2}\right) .
\end{aligned}
$$

The proof of observation error convergence is a particular case of the proof in Section 5.

Simulations results. Comparing the generalized phase plane of $x_{1}, x_{2}$ (system (2.2)) and $\hat{x}_{1}, \hat{x}_{2}$ (system (2.3) in dashed line) in Figure 2.2 with the generalized phase plane of $x_{1}, x_{2}$ (system (2.2)) and $\hat{x}_{1}, \hat{x}_{2}$ (system (2.4) in dashed line) in Figure 2.3, we note that the state of classical observer (system (2.3)) stays far from the real state longer than the 
16 Observability singularity in chaotic synchronization

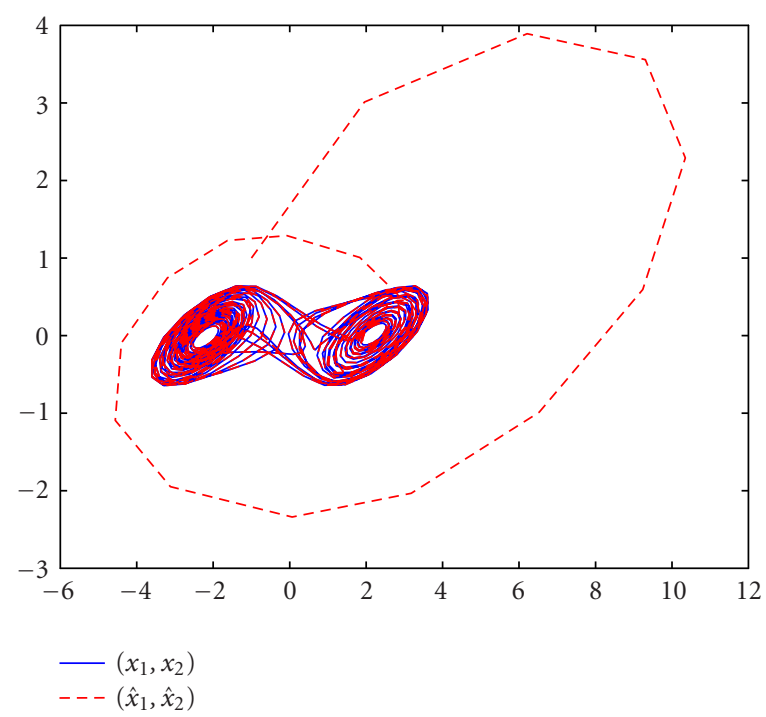

Figure 2.2. Double scroll attractor for systems (2.2) and (2.3).

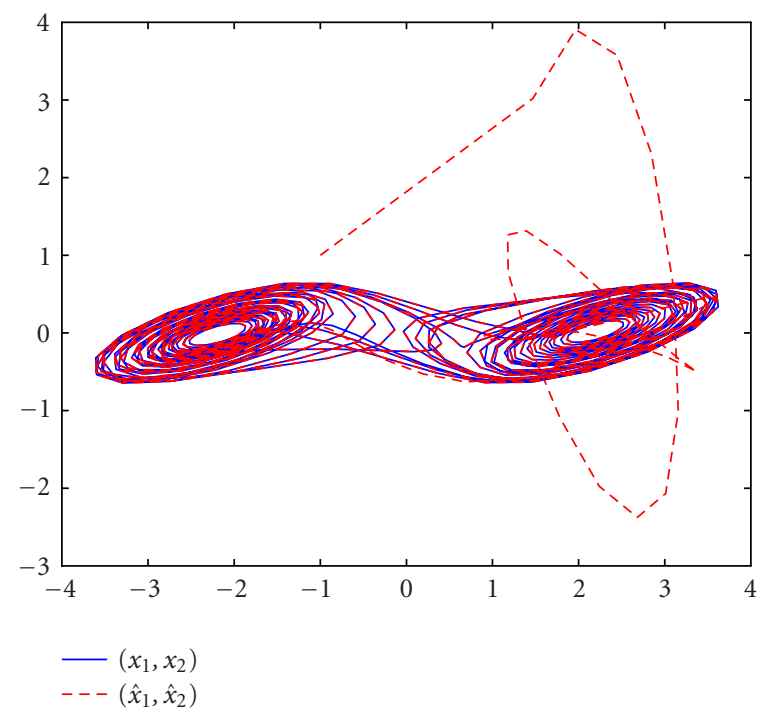

Figure 2.3. Double scroll attractor for systems (2.2) and (2.4).

state of the step-by-step observer (system (2.4)). This was confirmed by Figures 2.4 and 2.5 , where the observation error was shown, respectively, for the classical observer and the step-by-step observer. 




(a)

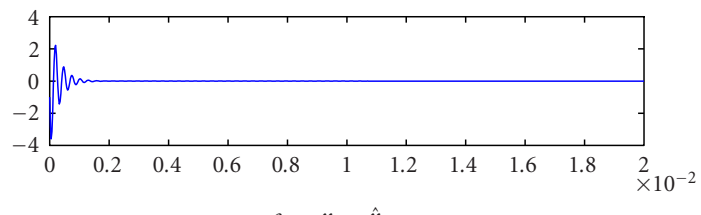

$e_{2}=x_{2}-\hat{x}_{2}$

(b)

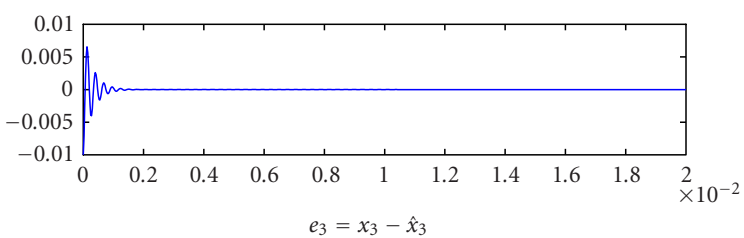

(c)

Figure 2.4. Observation error for systems (2.2) and (2.3).

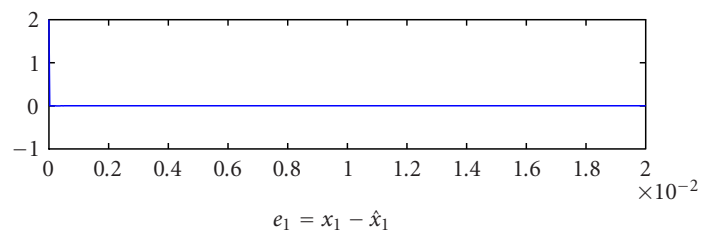

(a)

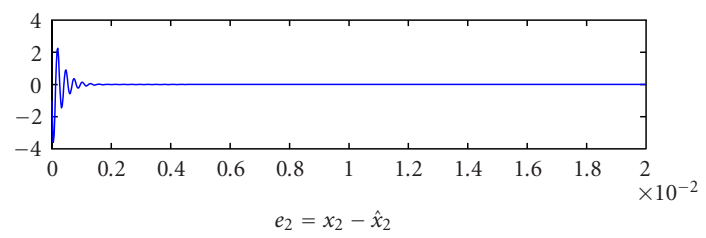

(b)

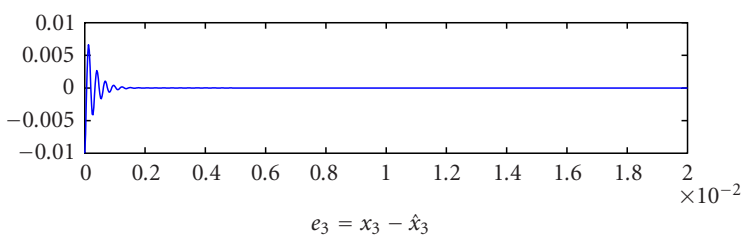

(c)

Figure 2.5. Observation error for systems (2.2) and (2.4). 
2.2. Nonlinearity of Chua circuit does not depend on the output. In $[3,18]$, a generalized output injection form was introduced, and following this way of thinking, a very interesting relation between a chaotic system and a generalized Hamiltonian system was done in [19]. Unfortunately, considering (2.2), with $x_{3}$ as the output instead of $x_{1}$, the nonlinearity is not an output function and the result about output injection (see $[3,18,19])$ cannot be used to design an observer.

Nevertheless, the observer matching condition (see [17]) was verified (i.e., the nonlinearity $f\left(x_{1}\right)$ is in $\operatorname{ker}(C, C A)$ (see Section 4$)$ ). Therefore, it is possible to design the following step-by-step sliding mode observer:

$$
\begin{aligned}
\frac{d \hat{x}_{1}}{d t} & =\frac{1}{C_{1}}\left(\frac{\tilde{x}_{2}-\hat{x}_{1}}{R}-f\left(\tilde{x}_{1}\right)\right)+E_{2} \lambda_{1} \operatorname{sign}\left(\tilde{x}_{1}-\hat{x}_{1}\right), \\
\frac{d \hat{x}_{2}}{d t} & =\frac{1}{C_{2}}\left(\frac{\hat{x}_{1}-\tilde{x}_{2}}{R}+x_{3}\right)+E_{3} \lambda_{2} \operatorname{sign}\left(\tilde{x}_{2}-\hat{x}_{2}\right), \\
\frac{d \hat{x}_{3}}{d t} & =\frac{1}{L}\left(-\hat{x}_{2}-R_{0} x_{3}\right)+\lambda_{3} \operatorname{sign}\left(x_{3}-\hat{x}_{3}\right), \\
y & =x_{3}
\end{aligned}
$$

with the following conditions: if $x_{3}=\hat{x}_{3}$, then $E_{3}=1$, else $E_{3}=0$, and if $\tilde{x}_{2}=\hat{x}_{2}$ and $E_{3}=1$, then $E_{2}=0$, else $E_{2}=0$. Moreover, by definition,

$$
\begin{aligned}
& \tilde{x}_{2}=\hat{x}_{2}-E_{3} L \lambda_{3} \operatorname{sign}\left(x_{3}-\hat{x}_{3}\right), \\
& \tilde{x}_{1}=\hat{x}_{1}+E_{2} C_{2} R \lambda_{2} \operatorname{sign}\left(\tilde{x}_{2}-\hat{x}_{2}\right) .
\end{aligned}
$$

The proof of observation error convergence will also be done in the same way of thinking as the proof of Section 5.

Simulations results. Figures 2.6 and 2.7 highlight the efficiency of the step-by-step observer for system (2.2) with $x_{3}$ as output. We note that the simulation results are very close to the previous one obtained with $x_{1}$ as output.

This section can be summarized in two points.

(i) Considering $x_{1}$ as output, using linearizable-by-output injection (see [13]), it is possible to observe the full state of Chua circuit.

(ii) Thanks to a step-by-step sliding mode observer, it is also possible to design a fullstate observer for Chua circuit with a new output $x_{3}$. For this output, the system is only observable but it is not linearizable by output injection.

In Sections 3 and 4, we want to do something different from this section and from the classical literature results. We will consider a system with observability bifurcation (i.e., the linear approximation is unobservable in one direction). To do this, we will recall, in Section 3, some new results on the observability bifurcation (see $[1,4]$ ). 


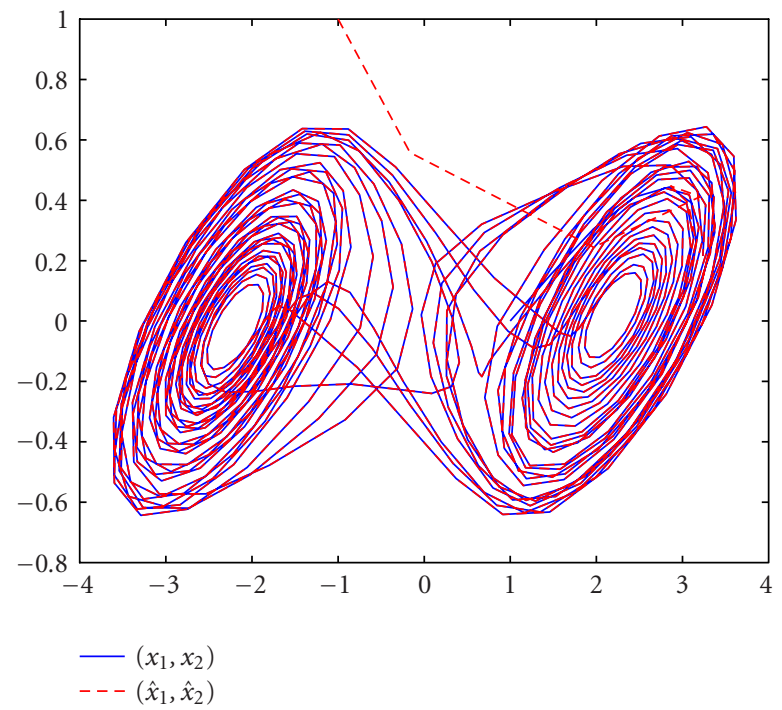

Figure 2.6. Double scroll attractor for systems (2.2) and (2.6).

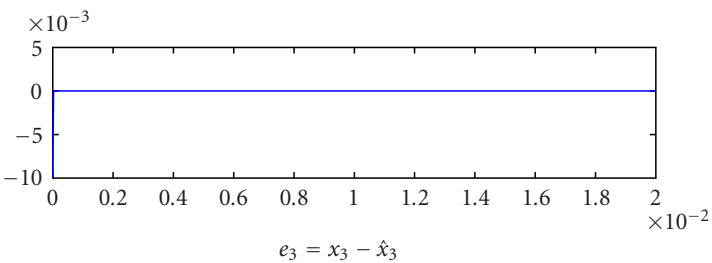

(a)



(b)

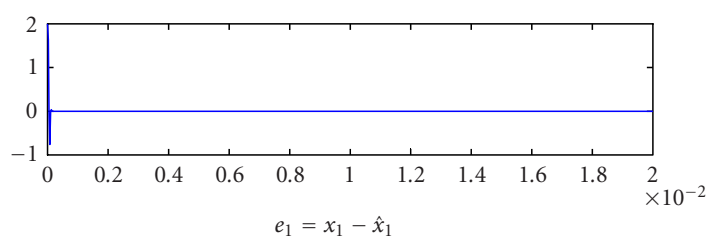

(c)

Figure 2.7. Observation error for systems (2.2) and (2.6). 


\section{Observability normal form}

Hereafter, we just recall one result of [4] for a nonlinear system with one real linear unobservable mode. This result is necessary to study the example of Section 2. This example was a transmission by synchronization of chaotic systems with observability bifurcation.

We consider a nonlinear single-input single-output (SISO) system:

$$
\dot{\xi}=f(\xi)+g(\xi) u, \quad y=C \xi
$$

where the vector fields $f, g: U \subset \mathbb{R}^{n} \rightarrow \mathbb{R}^{n}$ are assumed to be real-analytic and such that $f(0)=0$.

Setting $A=(\partial f / \partial \xi)(0)$ and $B=g(0)$ around the equilibrium point $\xi_{e}=0$, the system can be rewritten in the following form:

$$
\begin{aligned}
& \dot{z}=A z+B u+f^{[2]}(z)+g^{[1]}(z) u+O^{3}(z, u), \\
& y=C z
\end{aligned}
$$

where

$$
f^{[2]}(z)=\left(\begin{array}{c}
f_{1}^{[2]}(z) \\
f_{2}^{[2]}(z) \\
\vdots \\
f_{n}^{[2]}(z)
\end{array}\right), \quad g^{[1]}(z)=\left(\begin{array}{c}
g_{1}^{[1]}(z) \\
g_{2}^{[1]}(z) \\
\vdots \\
g_{n}^{[1]}(z)
\end{array}\right)
$$

with, for all $1 \leq i \leq n, f_{i}^{[2]}(z)$ and $g_{i}^{[1]}(z)$, respectively, homogeneous polynomials of degrees two and one in $z$ (see [11]).

In order to analyze the observability of system (3.1), we establish a quadratic equivalence, with respect to a quadratic diffeomorphism and output injection (which will be clarified below). The main drawback of this transformation is to preserve the observability properties. We first define the so-called quadratic equivalence.

Definition 3.1. System (3.2) is said to be quadratically equivalent modulo an output injection (QEMOI) to the system

$$
\begin{aligned}
& \dot{x}=A x+B u+\bar{f}^{[2]}(x)+\bar{g}^{[1]}(x) u+\beta^{[2]}(y)+\gamma^{[1]}(y) u+O^{3}(x, u), \\
& y=C x
\end{aligned}
$$

if there exist an output injection

$$
\beta^{[2]}(y)+\gamma^{[1]}(y) u
$$


and a diffeomorphism of the form

$$
x=z-\Phi^{[2]}(z)
$$

which carries $f^{[2]}(z)+g^{[1]}(z) u$ to $\bar{f}^{[2]}(x)+\bar{g}^{[1]}(x) u+\left(\beta^{[2]}(y)+\gamma^{[1]}(y) u\right)$, where

$$
\begin{aligned}
& \Phi^{[2]}(z)=\left(\Phi_{1}^{[2]}(z), \ldots, \Phi_{n}^{[2]}(z)\right)^{T} \\
& \beta^{[2]}(y)=\left(\beta_{1}^{[2]}(y), \ldots, \beta_{n}^{[2]}(y)\right)^{T},
\end{aligned}
$$

and, for all $1 \leq i \leq n, \Phi_{i}^{[2]}(z)$ and $\beta_{i}^{[2]}(y)$ are homogeneous polynomials in $z$, respectively, in $y$, of degree two, and $\gamma^{[1]}(y)=\left(\gamma_{1}^{[1]}(y), \ldots, \gamma_{n}^{[1]}(y)\right)^{T}$ with $\gamma_{i}^{[1]}(y)$ is homogeneous of degree one in $y$.

Assumption 3.2. The pair $(A, C)$ of system (3.1) has one unobservable real mode.

Under this assumption, there are a linear change of coordinates $(z=T \xi)$ and a Taylor expansion which transform system (3.1) into the following form:

$$
\begin{aligned}
& \dot{\tilde{z}}=A_{\mathrm{obs}} \tilde{z}+B_{\mathrm{obs}} u+\tilde{f}^{[2]}(z)+\tilde{g}^{[1]}(z) u+O^{3}(z, u), \\
& \dot{z}_{n}=\alpha z_{n}+\sum_{i=1}^{n-1} \alpha_{i} z_{i}+b_{n} u+f_{n}^{[2]}(z)+g_{n}^{[1]}(z) u+O^{3}(z, u), \\
& y=C_{\mathrm{obs}} \tilde{z}
\end{aligned}
$$

where $\tilde{z}=\left[z_{1}, z_{2}, \ldots, z_{n-1}\right]^{T}, z=\left[\tilde{z}^{T}, z_{n}\right]^{T}$,

$$
\begin{aligned}
A_{\mathrm{obs}} & =\left(\begin{array}{ccccc}
a_{1} & 1 & 0 & \cdots & 0 \\
a_{2} & 0 & 1 & \ddots & \vdots \\
\vdots & 0 & \vdots & \ddots & 0 \\
a_{n-2} & 0 & \cdots & 0 & 1 \\
a_{n-1} & 0 & \cdots & \cdots & 0
\end{array}\right), \\
B_{\mathrm{obs}} & =\left(\begin{array}{lll}
b_{1}, \ldots, b_{n-1}
\end{array}\right)^{T}, \\
C_{\mathrm{obs}} & =\left(\begin{array}{llll}
1 & 0 & \cdots & 0
\end{array}\right) .
\end{aligned}
$$

Remark 3.3. In this section, we deal with system with linearly observable part in the Brunovsky form (see [5]). Moreover, the output is always taken equal to the first state component. Consequently, the diffeomorphism $\left(x=z-\Phi^{[2]}(z)\right)$ is such that $\Phi_{1}^{[2]}(z)=0$. 
Proposition 3.4 [4]. System (3.2) is QEMOI to system (3.4) if and only if the following two homological equations are verified:

(i) $A \Phi^{[2]}(z)-\left(\partial \Phi^{[2]} / \partial z\right) A z=\bar{f}^{[2]}(z)-f^{[2]}(z)+\beta^{[2]}\left(z_{1}\right)$,

(ii) $-\left(\partial \Phi^{[2]} / \partial z\right) B=\bar{g}^{[1]}(z)-g^{[1]}(z)+\gamma^{[1]}\left(z_{1}\right)$, where

$$
\frac{\partial \Phi^{[2]}(z)}{\partial z} A z:=\left(\frac{\partial \Phi_{1}^{[2]}(z)}{\partial z} A z, \ldots, \frac{\partial \Phi_{n}^{[2]}(z)}{\partial z} A z\right)^{T}
$$

and $\partial \Phi_{i}^{[2]}(z) / \partial z$ is the Jacobian of $\Phi_{i}^{[2]}(z)$ for all $1 \leq i \leq n$.

Now, we can give the normal form associated to the QEMOI relation (see [4]).

THEOREM 3.5. There exists a quadratic diffeomorphism which transforms the quadratic part of system (3.8) into the quadratic normal form

$$
\begin{aligned}
\dot{x}_{1} & =a_{1} x_{1}+x_{2}+b_{1} u+\sum_{i=2}^{n} k_{1 i} x_{i} u+E_{1}, \\
\dot{x}_{2}= & a_{2} x_{1}+x_{3}+b_{2} u+\sum_{i=2}^{n} k_{2 i} x_{i} u+E_{2}, \\
\vdots & \\
\dot{x}_{n-2}= & a_{n-2} x_{1}+x_{n-1}+b_{n-2} u+\sum_{i=2}^{n} k_{(n-2) i} x_{i} u+E_{n-2}, \\
\dot{x}_{n-1}= & a_{n-1} x_{1}+b_{n-1} u+\sum_{j \geq i=2}^{n} h_{i j} x_{i} x_{j}+h_{1 n} x_{1} x_{n}+\sum_{i=2}^{n} k_{(n-1) i} x_{i} u+E_{n-1}, \\
\dot{x}_{n}= & \alpha_{n} x_{n}+\sum_{i=1}^{n-1} \alpha_{i} x_{i}+b_{n} u+\sum_{j=2}^{n} l_{1, j} x_{1} x_{j}+\sum_{j \geq i=2}^{n} l_{i, j} x_{i} x_{j}+\sum_{i=2}^{n} k_{n i} x_{i} u+E_{n},
\end{aligned}
$$

with $E_{i}=\beta_{i}^{[2]}(y)+\gamma_{i}^{[1]}(y) u$ for all $1 \leq i \leq n$.

The coefficients $h_{i, j}, l_{i, j}$, and $k_{i, i}$ for $2 \leq i \leq j \leq n$ are called quadratic normal numbers.

Remark 3.6. (1) We call the unobservability submanifold the subset $S_{n-1}$ of $U$ given by

$$
S_{n-1}=\left\{x \in U \mid \sum_{i=1}^{n-1} h_{i, n} x_{i}+2 h_{n, n} x_{n}+k_{(n-1), n} u=0\right\} .
$$

Thus, for a fixed input $u$, when system evolves on $S_{n-1}$ from the dynamics of $x_{n-1}$, we cannot recover any information on $x_{n}$ with the linear and quadratic terms.

(2) If $x \in S_{n-1}$ and $k_{n-1, n} \neq 0$, then with an appropriate change of input $u$ (universal input [8]), we can modify $S_{n-1}$ to locally restore the quadratic observability. Moreover, if $k_{n-1, n}=0$ and if there exists $i \in[1, n]$ such that $k_{i, n} \neq 0$, then the quadratic observability may be restored. 
(3) If $x \in S_{n-1}$ such that $u$ is not a function of $x_{n}$ and for all $i \in[1, n]$, we have $k_{i, n}=0$, then we use coefficient $\alpha_{n}$ to study the detectability propriety. For this, we distinguish three cases:

(a) if $\alpha_{n}<0$, then the state $x_{n}$ is detectable,

(b) if $\alpha_{n}>0$, then $x_{n}$ is unstable and consequently undetectable,

(c) if $\alpha_{n}=0$, we can use the center manifold theory in order to analyze the stability or instability of $x_{n}$ and consequently its detectability or undetectability.

Let $\Phi^{[2]}(x)=\left(0, \ldots, \Phi_{n}^{[2]}(x)\right)$ be the quadratic part of the diffeomorphism which gives Theorem 3.5. In the proof in [4, pages 2943-2944], $\Phi_{2}^{[2]}(x)$ is well determined from $f_{1}^{[2]}(x)$ and for all $3 \leq i \leq n-1, \Phi_{i}^{[2]}(x)$ is determined from $f_{i}^{[2]}(x)$ and the derivatives of $\Phi_{j}^{[2]}(x)$ and $f_{j}^{[2]}(x)$ for $2 \leq j \leq i-1$. However, on the last dynamic of the normal form (3.11), to compute resonant terms, we use Poincaré-Dulac theorem (see $[9,22]$ ) (from Poincaré-Dulac theorem, the condition for resonance is that there exists an eigenvalue $\lambda_{i}$ such that $\lambda_{i}=\sum_{j=1}^{n} k_{j} \lambda_{j}$ with $k_{j} \in N$ and $\left.\sum_{j=1}^{n} k_{j} \geq 2\right)$. So, it is interesting to use $\Phi_{n}^{[2]}(x)$ to cancel the quadratic terms in the last $\dot{x}_{n}$ dynamic; for this, we must check the following equation:

$$
\alpha_{n} \Phi_{n}^{[2]}(x)+\sum_{i=1}^{n-1} \alpha_{i} \Phi_{i}^{[2]}(x)=\frac{\partial \Phi_{n}^{[2]}}{\partial x} A_{\mathrm{obs}} x-f_{n}^{[2]}(x)+\beta_{n}^{[2]}\left(x_{1}\right)
$$

Unfortunately, the above equation is not fulfilled for arbitrary $\alpha_{n}$ and $\left(a_{i}\right)_{1 \leq i \leq n}$. Nevertheless, the conditions are less restrictive than the usual ones thanks to the output injection $\beta_{n}^{[2]}\left(x_{1}\right)$.

\section{Particular case: unknown input observer}

In this section, we propose to find a new application for the unknown input observer design. More precisely, we propose a new type of secure data transmission based on chaotic synchronization. For this, we have to recall and give some particular concepts of observer for a system with unknown input. On the basis of a concrete example, we put in evidence the ambivalence of certain differential models presenting discontinuity and unknown perturbation. This ambivalence often causes some errors of interpretation and this imposes specific tools simply evoked in this study. Nevertheless, it is possible with a step-by-step procedure to design an observer for such a system. Obviously, there are some restrictive conditions on the system to solve this problem (see [17, 23]).

We consider the nonlinear system

$$
\dot{\xi}=f(\xi)+p(\xi) w, \quad y=h(\xi),
$$

where $w \in \mathbb{R}$ is a bounded perturbation and vector fields $f, p: U \subset \mathbb{R}^{n} \rightarrow \mathbb{R}^{n}$ and $h: U \subset$ $\mathbb{R}^{n} \rightarrow \mathbb{R}$ are assumed to be real-analytic. Now, we can give a particular constraint in order 
to solve this problem. The unknown input observer design is locally solvable around $x=0$ for system (4.1) if the following two conditions are verified:

(i) $\operatorname{span}\left\{d h, d L_{f} h, \ldots, d L_{f}^{n-1} h\right\}$ is of rank $n$ at $\xi=0$,

(ii) $\left(\begin{array}{lllll}(d h)^{T} & \left(d L_{f} h\right)^{T} & \cdots & \left(d L_{f}^{n-1} h\right)^{T}\end{array}\right)^{T} p(\xi)=\left(\begin{array}{llll}0 & \cdots & 0 & *\end{array}\right)^{T}$,

where $p(\xi)=\left(\begin{array}{llll}0 & \cdots & 0 & p_{n}(\xi)\end{array}\right)^{T}$ and $*$ means a nonnull function almost everywhere in order to be able to identify the perturbation.

In order to reject the perturbation effect on the observer, conditions (i) and (ii) are called the observability matching condition.

Then, considering system (3.11) without input but with perturbation,

$$
\begin{aligned}
\dot{x}_{1} & =a_{1} x_{1}+x_{2}+E_{1}, \\
\dot{x}_{2} & =a_{2} x_{1}+x_{3}+E_{2}, \\
\vdots & \\
\dot{x}_{n-2} & =a_{n-2} x_{1}+x_{n-1}+E_{n-2}, \\
\dot{x}_{n-1} & =a_{n-1} x_{1}+\sum_{j \geq i=2}^{n} h_{i j} x_{i} x_{j}+h_{1 n} x_{1} x_{n}+E_{n-1}, \\
\dot{x}_{n} & =\alpha_{n} x_{n}+\sum_{i=1}^{n-1} \alpha_{i} x_{i}+\sum_{j=2}^{n} l_{1, j} x_{1} x_{j}+\sum_{j \geq i=2}^{n} l_{i, j} x_{i} x_{j}+p_{n}(x) w+E_{n} .
\end{aligned}
$$

With $E_{i}=\beta_{i}^{[2]}(y)$ for all $1 \leq i \leq n$, system (4.2) verifies the observability matching condition and it is a first generalization of our normal form, where we consider a specific perturbation.

Remark 4.1. For the sake of generality, hereafter $E_{i}(1 \leq i \leq n)$ is considered as $C^{0}$ function of the output. This less restrictive claim on $E_{i}$ changes partially our equivalent relation but allows us to consider the system as Chua circuit.

In fact, in Proposition 3.4, the nonquadratic term must be directly cancelled by the output injection.

In Section 5, we have to consider the step-by-step observer because it is, according to our knowledge, the only observer which works under observability matching condition, with $n \geqslant 2$.

\section{Transmission by inclusion method}

Now, in order to increase the security of transmission, we propose to add at the transmission by synchronization of a chaotic system some observability bifurcations. Here, we just give an illustrative example, so we consider again system (2.2) with $x_{1}$ as output but with $x_{4}=1 / L$ as a new state. The variation of $L$ is the information to pass on to the receiver. Moreover, we assume that there exist $K_{1}$ and $K_{2}$ such that $\left|x_{4}\right|<K_{1}$ and $\left|d x_{4} / d t\right|<K_{2}$. This means that the information signal and its variation are bounded. Thus, from these assumptions, we obtain the following system: 


$$
\begin{aligned}
\frac{d x_{1}}{d t} & =\frac{-1}{C_{1} R}\left(x_{1}-x_{2}\right)+\frac{f\left(x_{1}\right)}{C_{1}}, \\
\frac{d x_{2}}{d t} & =\frac{1}{C_{2} R}\left(x_{1}-x_{2}\right)+\frac{1}{C_{2}} x_{3}, \\
\frac{d x_{3}}{d t} & =-\left(x_{2}+R_{0} x_{3}\right) x_{4} \\
\frac{d x_{4}}{d t} & =\sigma \\
y & =x_{1}
\end{aligned}
$$

with $\sigma$ an unknown bounded function (i.e., $|\sigma|<K_{2}$ ).

This system has one unobservable real mode. Using the linear change of coordinates $z_{1}=x_{1}, z_{2}=x_{1} / C_{2} R+x_{2} / C_{1} R, z_{3}=x_{3} / C_{1} C_{2} R$, and $z_{4}=x_{4}$, we obtain

$$
\begin{aligned}
\frac{d z_{1}}{d t} & =\frac{-\left(C_{1}+C_{2}\right)}{C_{1} C_{2} R} z_{1}+z_{2}+\frac{f\left(z_{1}\right)}{C_{1}}, \\
\frac{d z_{2}}{d t} & =z_{3}+\frac{f\left(z_{1}\right)}{C_{1} C_{2} R}, \\
\frac{d z_{3}}{d t} & =\frac{z_{1} z_{4}}{C_{2}^{2} R}-\frac{z_{2} z_{4}}{C_{2}}-R_{0} z_{3} z_{4}, \\
\frac{d z_{4}}{d t} & =\sigma \\
y & =z_{1} .
\end{aligned}
$$

Equations (5.2) are on the observability normal form with $\alpha_{n}=0$ and resonant terms $h_{22}=h_{23}=0, h_{14}=1 / C_{2}^{2} R, h_{24}=1 / C_{2}$, and $h_{34}=-R_{0}$, but with $\sigma$ as a perturbation and a nonsmooth output injection $\left(f\left(z_{1}\right) / C_{1}, f\left(z_{1}\right) / C_{1} C_{2} R, 0,0\right)^{T}$. From Remark 3.6, we conclude that the resonant terms $h_{i 4} x_{i} x_{4}$ ensure the full-state local observability almost everywhere. The observability singularity is given by $z_{1} / C_{2}^{2} R-z_{2} / C_{2}-R_{0} z_{3}=0$, and taking into account this singularity, we can design an observer. Nevertheless, as system (5.1) has also a particular structure with $x_{4}=z_{4}$ and $x_{3}=C_{2} C_{1} R_{0} z_{3}$, we can design an observer directly on the original state (the physical one). Obviously, the observability singularity is the same, the equation $-x_{2}-R_{0} x_{3}=0$ is equivalent to $z_{1} / C_{2}^{2} R-z_{2} / C_{2}-R_{0} z_{3}=0$.

So, we will use the information contained in the terms $-x_{4} x_{2}-R_{0} x_{4} x_{3}$ in order to design a full-state observer and recover information on $x_{4}$ contained in the equation of $d x_{3} / d t$. For this, we use the following sliding mode observer:

$$
\begin{aligned}
\frac{d \hat{x}_{1}}{d t} & =\frac{1}{C_{1}}\left(\frac{\hat{x}_{2}-y}{R}-f(y)\right)+\lambda_{1} \operatorname{sign}\left(y-\hat{x}_{1}\right), \\
\frac{d \hat{x}_{2}}{d t} & =\frac{1}{C_{2}}\left(\frac{y-\tilde{x}_{2}}{R}+\hat{x}_{3}\right)+E_{1} \lambda_{2} \operatorname{sign}\left(\tilde{x}_{2}-\hat{x}_{2}\right), \\
\frac{d \hat{x}_{3}}{d t} & =\hat{x}_{4}\left(-\tilde{x}_{2}-R_{0} \tilde{x}_{3}\right)+E_{2} \lambda_{3} \operatorname{sign}\left(\tilde{x}_{3}-\hat{x}_{3}\right), \\
\frac{d \hat{x}_{4}}{d t} & =E_{3} \lambda_{4} \operatorname{sign}\left(\tilde{x}_{4}-\hat{x}_{4}\right), \\
y & =x_{1},
\end{aligned}
$$


with the following conditions: if $\hat{x}_{1}=x_{1}$, then $E_{1}=1$, else $E_{1}=0$; similarly if $\hat{x}_{2}=\tilde{x}_{2}$ and $E_{1}=1$, then $E_{2}=1$, else $E_{2}=0$; and finally if $\hat{x}_{3}=\tilde{x}_{3}$ and $E_{2}=1$, then $E_{3}=1$, else $E_{3}=0$. Moreover, in order to take into account the observability singularity $\left(\tilde{x}_{2}+R_{0} \tilde{x}_{3}=0\right)$, we set $E_{s}=1$ if $\tilde{x}_{2}+R_{0} \tilde{x}_{3} \neq 0$, else $E_{s}=0$. By definition, we take

$$
\begin{aligned}
& \tilde{x}_{2}=\hat{x}_{2}+E_{1} C_{1} R \lambda_{1} \operatorname{sign}\left(y-\hat{x}_{1}\right), \\
& \tilde{x}_{3}=\hat{x}_{3}+E_{2} C_{2} \lambda_{2} \operatorname{sign}\left(\tilde{x}_{2}-\hat{x}_{2}\right), \\
& \tilde{x}_{4}=\hat{x}_{4}-\frac{E_{3} E_{s}}{\left(\tilde{x}_{2}+R_{0} \tilde{x}_{3}-1+E_{S}\right)} \lambda_{3} \operatorname{sign}\left(\tilde{x}_{3}-\hat{x}_{3}\right) .
\end{aligned}
$$

Sketch of proof. In this sketch of proof, we implicitly assume that system (5.1) has a bounded state (i.e., obvious due to energy consideration). Consequently, in the observer, we add saturation on the integrator in order to have also a bounded state observer. From these two boundless considerations, all $\lambda_{i}$ may be easily chosen as constants (see [21]).

Step 1. Assuming that $E_{1}=0$ (if $E_{1}=1$, we directly move to the next step), the observation error dynamics $(e=x-\hat{x})$ is

$$
\begin{aligned}
\frac{d e_{1}}{d t} & =\frac{e_{2}}{C_{1} R}-\lambda_{1} \operatorname{sign}\left(x_{1}-\hat{x}_{1}\right), \\
\frac{d e_{2}}{d t} & =\frac{e_{2}}{C_{2} R}+\frac{e_{3}}{C_{2}}, \\
\frac{d e_{3}}{d t} & =\left[x_{4}\left(-x_{2}-R_{0} x_{3}\right)\right]-\left[\hat{x}_{4}\left(-\hat{x}_{2}-R_{0} \hat{x}_{3}\right)\right], \\
\frac{d e_{4}}{d t} & =0 .
\end{aligned}
$$

Due to the finite-time convergence of the sliding mode, there exists $\tau_{1} \geq 0$ such that for all $t \geq \tau_{1}, \hat{x}_{1}=x_{1}$ and we pass to the following step.

Step 2. As $\hat{x}_{1}=x_{1}$, then $E_{1}=1$, and as $e_{1}=0$ for all $t \geq \tau_{1}$, then $\dot{e}_{2}=0$, and consequently, invoking the equivalent vector $[21], \tilde{x}_{2}=x_{2}$ and we obtain

$$
\begin{aligned}
\frac{d e_{1}}{d t} & =\frac{e_{2}}{C_{1} R}-\lambda_{1} \operatorname{sign}\left(x_{1}-\hat{x}_{1}\right)=0, \\
\frac{d e_{2}}{d t} & =\frac{e_{3}}{C_{2}}-\lambda_{2} \operatorname{sign}\left(x_{2}-\hat{x}_{2}\right), \\
\frac{d e_{3}}{d t} & =\left[x_{4}\left(-x_{2}-R_{0} x_{3}\right)\right]-\left[\hat{x}_{4}\left(-\hat{x}_{2}-R_{0} \hat{x}_{3}\right)\right], \\
\frac{d e_{4}}{d t} & =0 .
\end{aligned}
$$

Due to the finite-time convergence of the sliding mode, there exists $\tau_{2} \geq \tau_{1} \geq 0$ such that for all $t \geq \tau_{2}, \hat{x}_{2}=\tilde{x}_{2}=x_{2}$ and we pass to the following step. 
Step 3. As $\hat{x}_{2}=x_{2}$ and $E_{1}=1$, then $E_{2}=1$, and as $e_{2}=0$ for all $t \geq \tau_{2}$, then $\dot{e}_{3}=0$, and consequently, invoking the equivalent vector, $\tilde{x}_{3}=x_{3}$ and we obtain

$$
\begin{aligned}
\frac{d e_{1}}{d t} & =\frac{e_{2}}{C_{1} R}-\lambda_{1} \operatorname{sign}\left(x_{1}-\hat{x}_{1}\right)=0, \\
\frac{d e_{2}}{d t} & =\frac{e_{3}}{C_{2}}-\lambda_{2} \operatorname{sign}\left(x_{2}-\hat{x}_{2}\right)=0, \\
\frac{d e_{3}}{d t} & =-\left(x_{2}+R_{0} x_{3}\right) e_{4}-\lambda_{3} \operatorname{sign}\left(x_{3}-\hat{x}_{3}\right), \\
\frac{d e_{4}}{d t} & =0 .
\end{aligned}
$$

Due to the finite-time convergence of the sliding mode, there exists $\tau_{3} \geq \tau_{2} \geq \tau_{1} \geq 0$ such that for all $t \geq \tau_{3}, \hat{x}_{3}=\tilde{x}_{3}=x_{3}$ and we pass to the following step.

Step 4. As $\hat{x}_{3}=x_{3}$ and $E_{3}=3$, then $E_{3}=1$ and we obtain

$$
\begin{aligned}
& \frac{d e_{1}}{d t}=\frac{e_{2}}{C_{1} R}-\lambda_{1} \operatorname{sign}\left(x_{1}-\hat{x}_{1}\right)=0, \\
& \frac{d e_{2}}{d t}=\frac{e_{3}}{C_{2}}-\lambda_{2} \operatorname{sign}\left(x_{2}-\hat{x}_{2}\right)=0, \\
& \frac{d e_{3}}{d t}=-\left(x_{2}+R_{0} x_{3}\right) e_{4}-\lambda_{3} \operatorname{sign}\left(x_{3}-\hat{x}_{3}\right)=0, \\
& \frac{d e_{4}}{d t}=E_{s} \lambda_{4} \operatorname{sign}\left(\tilde{x}_{4}-\hat{x}_{4}\right) .
\end{aligned}
$$

Therefore, if $E_{s}=1$, then $e_{4}$ goes to zero in finite time, else $E_{s}=0$ and we freeze the $e_{4}$ dynamic (the data acquisition). Nevertheless, the singularity $\left(x_{2}+R_{0} x_{3}\right)$ is local, so as the transmitter is chaotic, we never stay enough time on the singularity to alter substantially the data acquisition.

In order to illustrate the efficiency of the method, we chose to transmit the following message:

$$
L(t)=L+0.1 \sin (100 t) \quad \text { with } L=18.8 \mathrm{mH} .
$$

Simulations results. In Figure 5.2, if we set $E_{s}=0$ on a big neighborhood of the singularity manifold $\left(x_{2}+R_{0} x_{3}\right)$, we lose for a long time the information on $x_{4}$. We notice that the convergence of the state $\hat{x}_{4}$ of the observer towards $x_{4}$ of the system of origin (5.1) depends on the choice of $E_{s}$. In order to get back all the information on $x_{4}$, it is necessary to take $E_{s}=0$ on a very small neighborhood of the singularity manifold $\left(x_{2}+R_{0} x_{3}\right)$, as we notice it on Figure 5.4, contrary to the first two which were realized with a too broad neighborhood. And we see the double scroll of the transmitter and the receiver which are completely synchronized. 


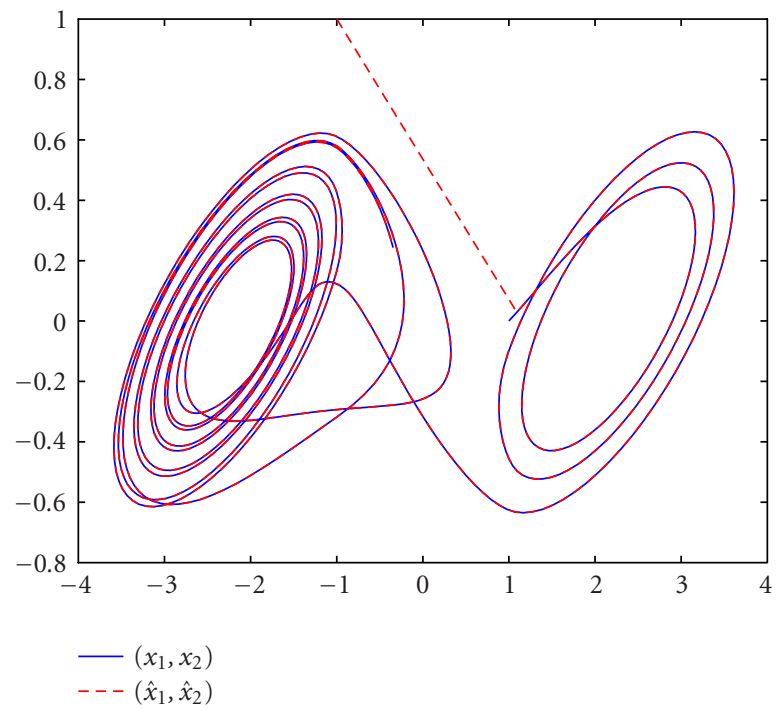

Figure 5.1. Double scroll attractor for systems (5.1) and (5.3), with $E_{s}=0$ if $\left(x_{2}+R_{0} x_{3}\right) \in$ ]$-0.01,0.01[$.
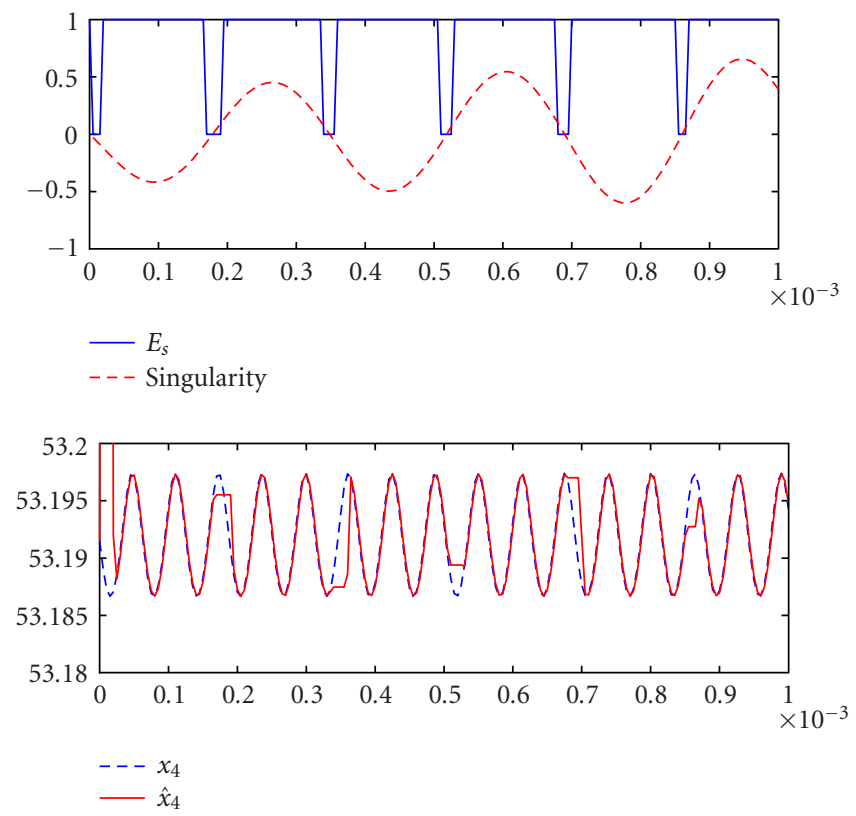

Figure 5.2. $x_{4}, \hat{x}_{4}, E_{s}$, and the singularity $\left(x_{2}+R_{0} x_{3}\right)$, with $E_{s}=0$ if $\left.\left(x_{2}+R_{0} x_{3}\right) \in\right]-0.01,0.01[$. 


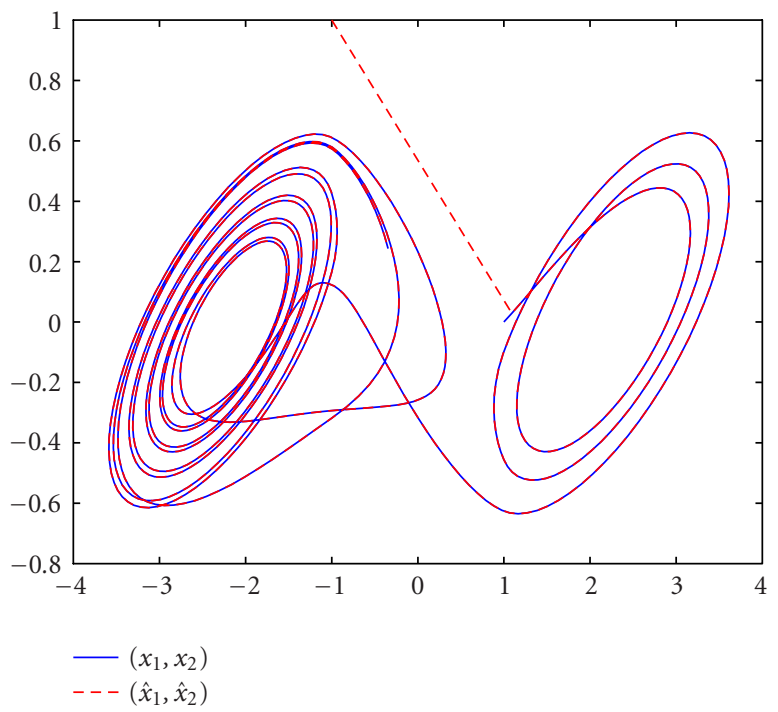

Figure 5.3. Double scroll attractor for systems (5.1) and (5.3), with $E_{s}=0$ if $\left(x_{2}+R_{0} x_{3}\right) \in$ ]$-0.0001,0.0001[$.
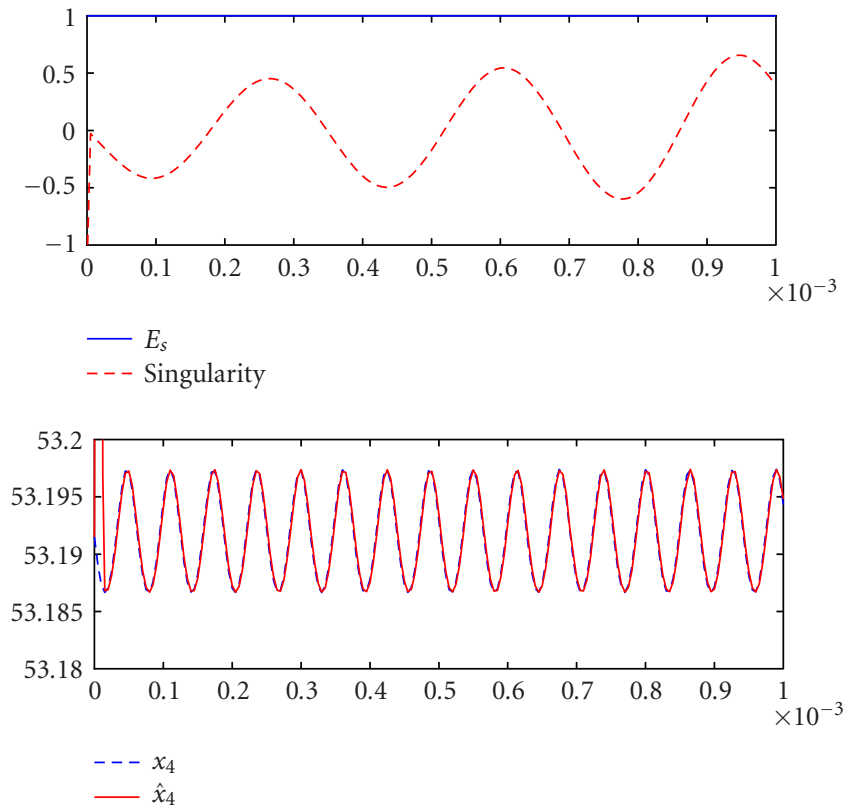

Figure 5.4. $x_{4}, \hat{x}_{4}, E_{s}$, and the singularity $\left(x_{2}+R_{0} x_{3}\right)$, with $E_{s}=0$ if $\left.\left(x_{2}+R_{0} x_{3}\right) \in\right]-0.0001,0.0001[$. 
Remark 5.1. In practice, we add some lowpass filter on the auxiliary state $\tilde{x}_{i}$ and we set $E_{i}=1$ for $i \in\{1,2,3\}$, not exactly when we are on the sliding surface but when we are close enough. Similarly, $E_{s}=0$ when we are close to the singularity, not only when we are on.

\section{References}

[1] J. P. Barbot, I. Belmouhoub, and L. Boutat-Baddas, Observability normal forms, New Trends in Nonlinear Dynamics and Control, and Their Applications (W. Kang, M. Xiao, and C. Borges, eds.), Lecture Notes in Control and Inform. Sci., vol. 295, Springer, Berlin, 2003, pp. 3-17.

[2] J. P. Barbot, T. Boukhobza, and M. Djemai, Sliding mode observer for triangular input form, Proc. 35th IEEE Conference on Decision and Control, Kobe, 1996, pp. 1489-1490.

[3] G. Besançon, A viewpoint on observability and observer design for nonlinear systems, New Directions in Nonlinear Observer Design (Geiranger Fjord, 1999) (H. Nimeijer and T. I. Fossen, eds.), Lecture Notes in Control and Inform. Sci., vol. 244, Springer-Verlag, London, 1999, pp. 3-22.

[4] L. Boutat-Baddas, D. Boutat, J. P. Barbot, and R. Tauleigne, Quadratic observability normal form, Proc. 40th IEEE Conference on Decision and Control, Florida, 2001, pp. 2942-2947.

[5] P. Brunovský, A classification of linear controllable systems, Kybernetika (Prague) 6 (1970), 173188.

[6] G. Chen and X. Dong, From Chaos to Order. Methodologies, Perspectives and Applications, World Scientific Series on Nonlinear Science. Series A: Monographs and Treatises, vol. 24, World Scientific Publishing, New Jersey, 1998.

[7] K. M. Cuomo, A. V. Oppenheim, and S. H. Strogatz, Synchronization of Lorenz-based chaotic circuit with application to communications, IEEE Trans. Circuits and Systems 40 (1993), 626633.

[8] J.-P. Gauthier and G. Bornard, Observability for any $u(t)$ of a class of nonlinear systems, IEEE Trans. Automat. Control 26 (1981), no. 4, 922-926.

[9] P. Glendinning, Stability, Instability and Chaos: an Introduction to the Theory of Nonlinear Differential Equations, Cambridge Texts in Applied Mathematics, Cambridge University Press, Cambridge, 1994.

[10] R. Hermann and A. J. Krener, Nonlinear controllability and observability, IEEE Trans. Automat. Control 22 (1977), no. 5, 728-740.

[11] W. Kang and A. J. Krener, Extended quadratic controller normal form and dynamic state feedback linearization of nonlinear systems, SIAM J. Control Optim. 30 (1992), no. 6, 1319-1337.

[12] P. V. Kokotović, R. E. O'Malley Jr., and P. Sannuti, Singular perturbations and order reduction in control theory - an overview, Automatica J. IFAC 12 (1976), no. 2, 123-132.

[13] A. J. Krener and A. Isidori, Linearization by output injection and nonlinear observers, Systems Control Lett. 3 (1983), no. 1, 47-52.

[14] A. J. Krener and M. Q. Xiao, Nonlinear observer design in the Siegel domain, SIAM J. Control Optim. 41 (2002), no. 3, 932-953.

[15] H. Nijmeijer and I. M. Y. Mareels, An observer looks at synchronization, IEEE Trans. Circuits Systems I Fund. Theory Appl. 44 (1997), no. 10, 882-890.

[16] U. Parlitz, L. O. Chua, L. j. Kocarev, K. S. Halle, and A. Shang, Transmission of digital signals by chaotic synchronization, Internat. J. Bifur. Chaos Appl. Sci. Engrg. 2 (1992), no. 4, 973-997.

[17] W. Perruquetti and J. P. Barbot, Sliding Mode Control in Engineering, Marcel Dekker, Oregon, 2002.

[18] F. Plestan and A. Glumineau, Linearization by generalized input-output injection, Systems Control Lett. 31 (1997), no. 2, 115-128. 
[19] H. Sira-Ramirez and C. Cruz-Hernandez, Synchronization of chaotic systems: a generalized Hamiltonian systems approach, Internat. J. Bifur. Chaos Appl. Sci. Engrg. 11 (2001), no. 5, 1381-1395.

[20] A. N. Tikhonov, Systems of differential equations containing small parameters multiplying the derivative, Mat. Sb. (N.S.) 31 (1952), 575-586 (Russian).

[21] V. I. Utkin, Sliding Modes in Control and Optimization, Communications and Control Engineering Series, Springer-Verlag, Berlin, 1992.

[22] S. Wiggins, Introduction to Applied Nonlinear Dynamical Systems and Chaos, Texts in Applied Mathematics, vol. 2, Springer-Verlag, New York, 1990.

[23] Y. Xiong and M. Saif, Sliding mode observer for nonlinear uncertain systems, IEEE Trans. Automat. Control 46 (2001), no. 12, 2012-2017.

[24] T. Yang and L. Chua, Secure communication via chaotic parameter modulation, IEEE Trans. Circuits Systems I Fund. Theory Appl. 43 (1996), no. 9, 817-819.

L. Boutat-Baddas: Equipe Commande des Systèmes, École Nationale Supérieure de l'Electronique et de ses Applications, 6 avenue du Ponceau, 95014 Cergy Cedex, France

E-mail address: boutat@ensea.fr

J. P. Barbot: Equipe Commande des Systèmes, École Nationale Supérieure de l'Electronique et de ses Applications, 6 avenue du Ponceau, 95014 Cergy Cedex, France

E-mail address: barbot@ensea.fr

D. Boutat: Laboratoire Vision et Robotique, École Nationale Supérieure d'Ingenieurs de Bourges et Université d'Orléans, 10 Boulevard Lahitolle, 18020 Bourges Cedex, France

E-mail address: driss.boutat@ensi-bourges.fr

R. Tauleigne: Equipe Commande des Systèmes, École Nationale Supérieure de l'Electronique et de ses Applications et Conservatoire National de Arts et Métiers, 6 avenue du Ponceau, 95014 Cergy Cedex, France

E-mail address: tauleign@ensea.fr 




Advances in

Operations Research

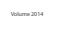

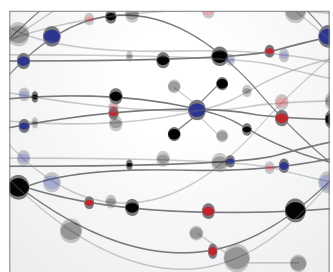

\section{The Scientific} World Journal
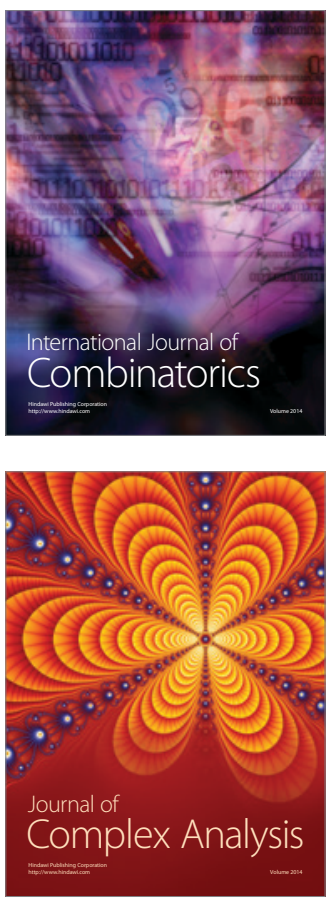

International Journal of

Mathematics and

Mathematical

Sciences
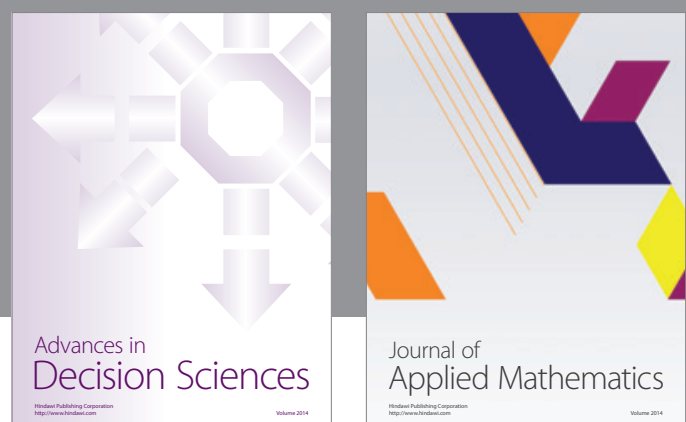

Journal of

Applied Mathematics
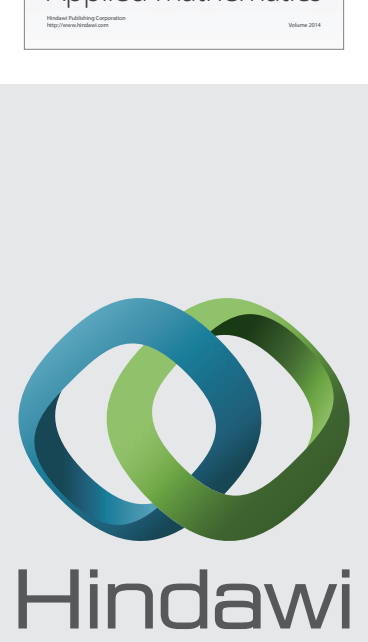

Submit your manuscripts at http://www.hindawi.com
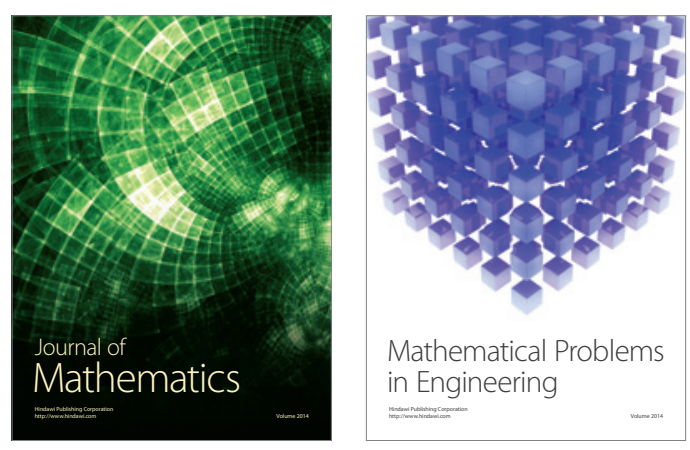

Mathematical Problems in Engineering
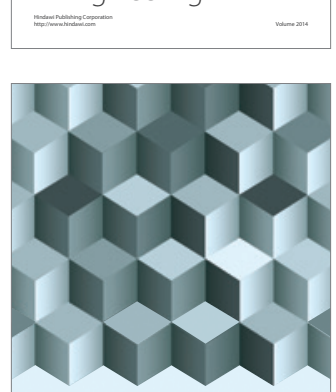

Journal of

Function Spaces
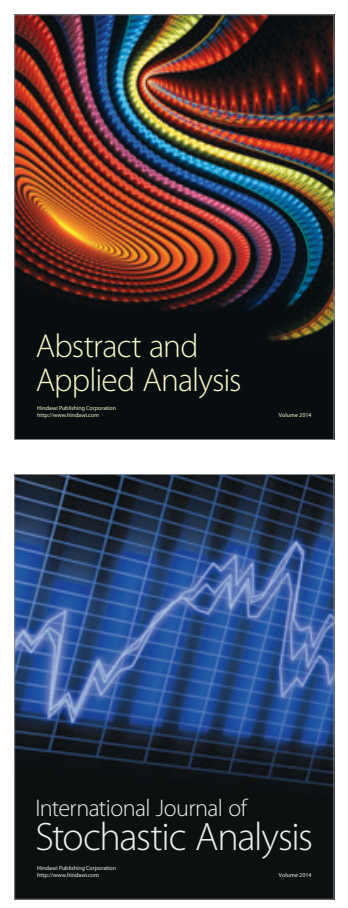

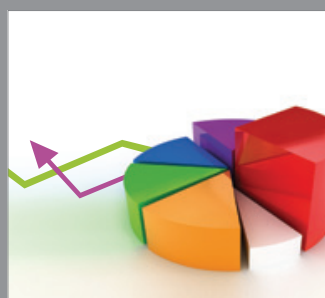

ournal of

Probability and Statistics

Promensencen
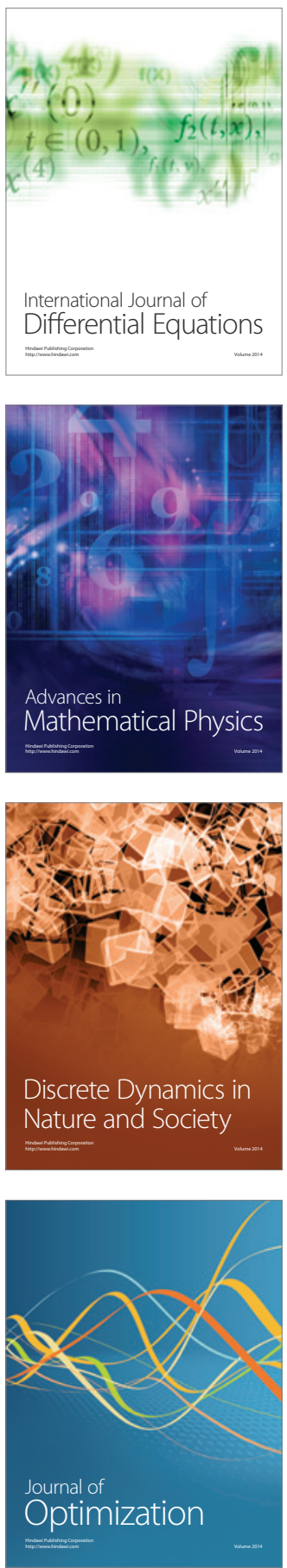